\title{
Complex-Network-Based Cyber-Physical Production Systems Subject to Cascading Failures
}

\author{
Sichao LIU ${ }^{\mathrm{a}}$, Lihui WANG ${ }^{\mathrm{a}, 1}, \mathrm{Xi}$ Vincent WANG ${ }^{\mathrm{a}}$ and Magnus WIKTORSSON ${ }^{\mathrm{b}}$ \\ ${ }^{a}$ Department of Production Engineering, KTH Royal Institute of Technology, 10044 Stockholm, Sweden \\ ${ }^{\mathrm{b}}$ Department of Sustainable Production Development, KTH Royal Institute of Technology, 15181 Södertälje,
}

Sweden

\begin{abstract}
The manufacturing industry is facing multi-dimensional, ever-growing challenges ranging from the lack of real-time manufacturing resource data, the inability of catching production exceptions, to the occurrence of cascading failures. This paper proposes a network-based cyber-physical production system to model, diagnose and control complex production systems subject to cascading failures. The goal is to study and characterise the evolution of cascading failure mechanisms and further mitigate the vulnerability of the manufacturing system. This is achieved through the deployment and synergistic integration of the Internet of technology with the reliability importance theory. The paper contributes to the network reliability theory and applications by proposing new importance measures and strategies to support the operation of cyber-physical production systems.
\end{abstract}

Keywords. Cyber-physical production systems, complex network, cascading failure, reliability importance, production network

\section{Introduction}

Current manufacturing systems are required to establish flexible, adaptive, and reliable manufacturing operations locally and globally by using integrated information communication technology (ICT) and smart algorithms [1]. Manufacturing systems depends on the timely acquisition, distribution, and utilisation of real-time data from both machines and processes on manufacturing shop floors. Dynamic data flow and integration of the computational entity of the manufacturing systems and the physical world plays an important role in guaranteeing good performance [2]. Cyber-physical systems (CPSs) show a promising potential working as the solution to the needs of the current manufacturing systems [3], which is an essential concept among smart manufacturing technologies. The term of CPS was initialised in the US in 2006 [4] and is composed of collaborating computational entities that connect the cyber world with the surrounding physical environments or processes through data access in an Internet environment, i.e. physical processes are monitored and controlled by the embedded computers and feedbacks loops are designed to allow both sides to affect each other. CPS also positively affected manufacturing and production by introducing cyber-physical production systems (CPPS) in process automation and control [5]. CPPS is a

\footnotetext{
${ }^{1}$ Corresponding Author. [lihui.wang@iip.kth.se]
} 
manufacturing-centred concept from CPS that fuses computer science, information and communication technology, and manufacturing engineering. The introduction of a CPPS allows smart manufacturing that aids in various decision-making processes by predicting the future based on past and present situations [6]. One industrial application of CPPS in the production engineering is a holonic cyber-physical production system used for the remote monitoring and control of machine tool, which uses the combined strength of holons, agents and function blocks [7].

However, current manufacturing systems are characterised by complex product structures, huge multi-source heterogeneous manufacturing resources, and random uncertainty factors of production and these typical characteristics of the manufacturing systems result in the lack of real-time information of manufacturing resources, production exception, and cascading failures [8]. Complex relations between production units in the manufacturing system and the topological structure make it difficult to build a cyber-physical-enabled network structure. The propagation and diffusion of failures lead to the malfunction of other units and the entire manufacturing network is broken down.

New paradigms and technologies such as the Internet of Things (IoT), complex network theory, and reliability importance, have recently gained a lot of attention in the manufacturing fields because of these challenges. They can be applied as a solution to model complex-network-based CPPS. Therefore, the research questions arising from the design of complex-network-based CPPS are summarised as:

1. How to sense and capture real-time information of manufacturing resources, catch production exceptions, and monitor cyber-physical production systems?

2. How to build a network-based cyber-physical production system model, and identify the cascading failures based on local load preferential redistribution rules?

3. How to monitor and derive the evolutionary mechanism of the importance of nodes within a cascading failure?

Real-time data acquisition for manufacturing systems is a prerequisite of carrying on the smart decision-making on the manufacturing operation. IoT, as a core technology in CPPS, refers as a wireless communication capability integrated with sensors and computing that allows the collection of data related to uniquely identifiable objects through the internet [9][10]. A typical architecture of IoT is composed of four layers: sensing layer, networking layer, middleware layer, and application layer. The sensing layer is responsible for sensing and capturing the real-time information of resources, devices, and further sharing among the identified units through a constructed wireless network with tags and sensors [11]. RFID (radio frequency identification), as a key enabling technology of IoT, allows microchips to transmit the identification information to a reader through wireless communication [12], and the main function of an application layer is to integrate the methodologies and functions of the system to achieve IoTenabled industrial applications. For example, an IoT-enabled real-time data collection framework was developed for shop-floor production performance analysis for the manufacturing systems [13]. An IoT-enabled manufacturing system, which is a multisource real-time data-driven manufacturing system, is developed for monitoring, decision making and management from the production orders assigned to the required work-in-progress or finished products [14]. 
The manufacturing system can be defined as a manufacturing network from a perspective of graph theory [15]. A complex manufacturing system consists of a great number of manufacturing elements, workstations, machines, buffers, transportation devices [16], while these physical objects and cyber entities within the manufacturing system can be considered as the nodes of the network, and the relation between them can be defined as edges. In addition, the degree of a node is the number of edges that are incident to the node. Due to the high complexity of the internal elements within the network, local tiny disturbance in the network such as exceptions and failures can induce internal cascading failure. The spread of the failures in the network can also lead to serious consequences and largely affect the performance of the manufacturing systems [17]. The load-capacity model in the cascading failure model has gained a lot of attention because of the flow transmission within all the physical or cyber networks. The network is also proven with overload issues. The Motter-Lai (ML) capacity model studied the overload-based cascading failure in the network and assumed that the original load of a node is proportional to the capacity of the node. Then, the improved model was developed and concluded that the node with a high original load can burden more load when happening overload issues [18]. The model proposed in [19] also shown that the capacity of the node is related to the original load and degree of the node. For these proposed models, the load of the network was instantaneously adjusted and adapted into the new status of the network after the node failure. This requires all the nodes in the network have the global information of the network.

The rest of the paper is organised as follows. Section 1 describes the framework of complex-network-enabled cyber-physical production systems. Section 2 presents the complex network modelling of the cyber-physical production system. Section 3 explains the evolutionary mechanism of the cyber-physical-based complex network within the cascading failure. Finally, Section 4 draws conclusions and highlights future work.

\section{A complex-network-enabled cyber-physical production system}

In this section, the architecture of the complex-network-enabled CPPS is shown as Figure. 1. It consists of a "cyber" part and a "physical" part and four modules, namely execution part (physical devices) module, event-driven data sensing/processing module, complex-network-based CPPS module, and human user module. Under this architecture, real-time data of devices in the physical shop floor with an embedded sensor network is sensed and collected. The data is transmitted and shared with the computational entity of the system via a network. The computational entity is responsible for data processing, monitoring and controlling the action of the physical entities. In a cyber part, the complex network of CPPS is developed. Humans as the third part of the system can then interact with the system when necessary/desired through embedded interfaces.

In the context of CPPS, the physical part presents the actual physical equipment on the shop floor and the sensed data collected by the embedded sensor network are processed and defined as basic, complex, and key events. The definitions of the basic, complex, and key events are given in [20]. The event-driven information collection system consists of physical sensing, data transmission, information processing, and data storage from the bottom to the top. The physical sensing module is used to sense and transmit the information of the production tasks and monitor the production status of the devices through the embedded sensors and RFID devices. Under the built-in wireless network and embedded transmission devices, a data transmission module is used to 


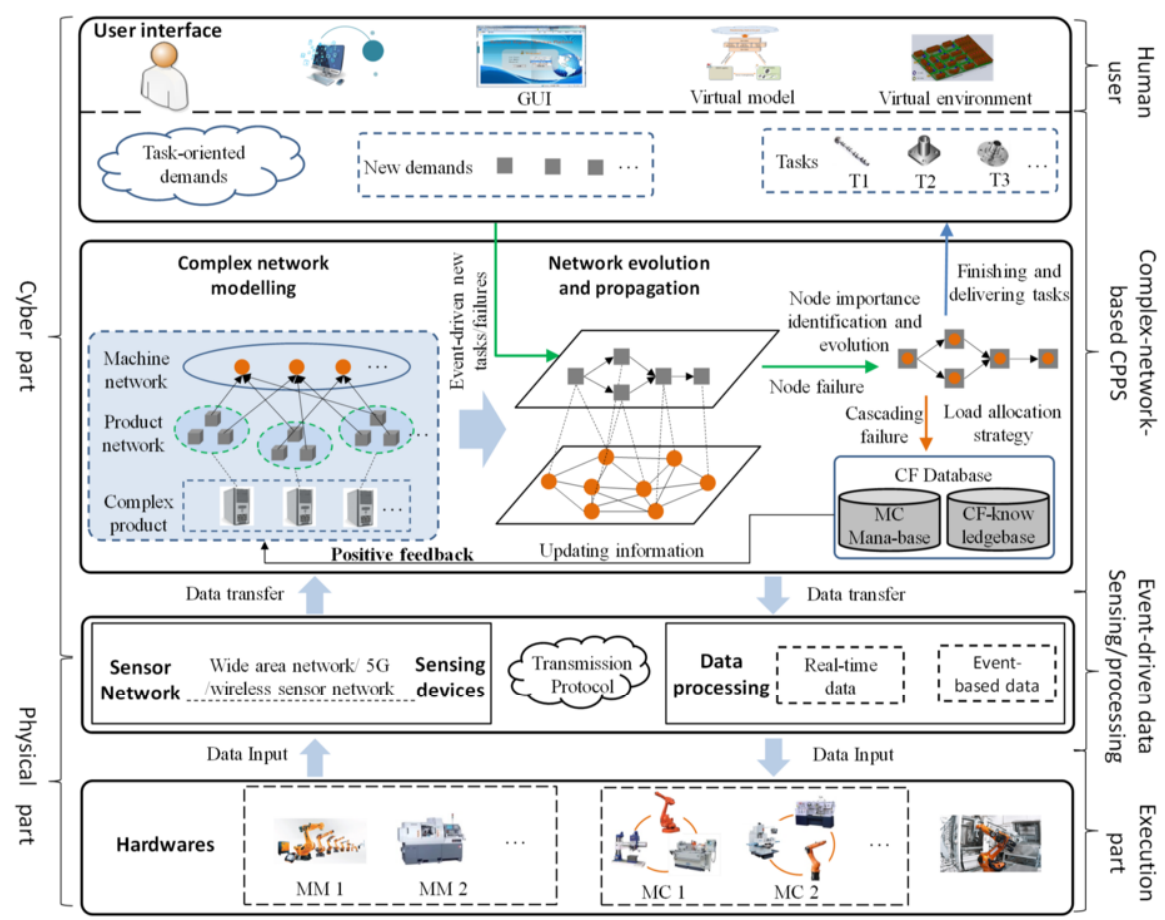

Figure 1. A complex-network-based cyber-physical production system

transmit data to the information processing module, which can identify the production exception, and achieve the added value of the information through data filtering and definition. According to the definition of the events collected from the physical shop floor, these event-based data are defined and classified. They are machine key data, product key data, system exception, and network node failures. The system exception includes the exceptions from the product network and machine network.

\section{Complex network modelling of CPPS}

Based on real-time data of the manufacturing resources, two local network models of CPPS are developed, namely a machine network model and a product network model. The correlation of the required machining process of products and the machining capacity of machines establishes a complex internal connection of the local networks.

\subsection{Machine network model}

The basic event in the machine network can be considered as a basic unit that represents the physical properties of the nodes of the machine network. A key event represents the change of the whole performance of the machine network and the change derives from the change of the node's status of the machine network. Meanwhile, this also leads to the change of the node's topology. The physical properties and topology of the nodes of the machine network keep stable in a steady stage, and the structure of the network also does 
not change. The degree of the node in the machine network is defined as the importance of the nodes and is determined by the connection relation of the node and its attributes. The topology of the network changes with the status of the nodes. Therefore, the evolutionary mechanism of the network plays an important role in the change of network. The two load adjustment of the network nodes namely cut edge and reconnection, is the key of the evolutionary mechanism of the machine network.

Figure 2(a) shows the relation of the machines used for machining a part and the corresponding machine network model. In a certain time interval, the topology and node's status of the network keep stable. When the system enters into a new time interval and production tasks on the production devices change, then the topology of the network and the status of machines change accordingly. Once the current machine does not have the ability of handling new tasks, a new node with the capability of producing new tasks joins the machine network. Meanwhile, the new node builds a connection with other nodes. Therefore, the number of the nodes and the edges of the network increase. In the context of network evolution, the exceptions such as overload, failure, machining breakdown, and part damage, lead to disconnection and reconnection of the nodes in the machine network. When reconnecting with other nodes, the desired high robustness of the network makes the node connect with nodes with large degrees. The nodes with large degrees have a stronger ability of handling exceptions. This behaviour is defined as the preferential attachment.

\subsection{Product network model}

From the above discussion, it is concluded that the production of complex products has an obvious hierarchical relation. It is because a complex product is composed of various components which are assembled by several parts, while the parts are completed by different machining processes. Therefore, a hierarchical product network is developed in Figure 2(b) which consists of the process-level (first-order) network, the part-level (second-order) network, the component-level (third-order) network, and product-level (fourth-order) network. The production process is considered as a basic unit in the context of the complex product production, and the relation between various production process is highly complex. According to the hierarchical structure of the product network, a part in the first-order network is finished by several production processes. This machining process is formed by the serial production relation. The second-order network is composed of the part-level tasks and the fourth-order network is the product-level tasks, which is composed of the component-level tasks (the third-order network).

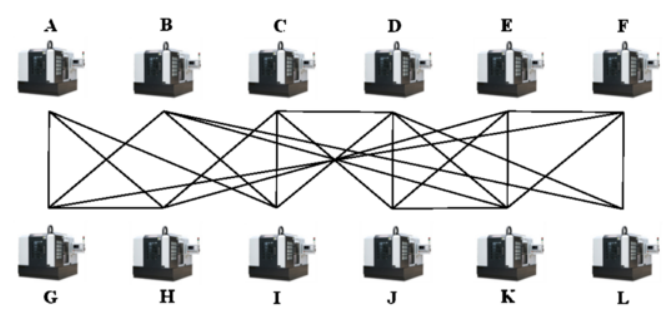

(a)

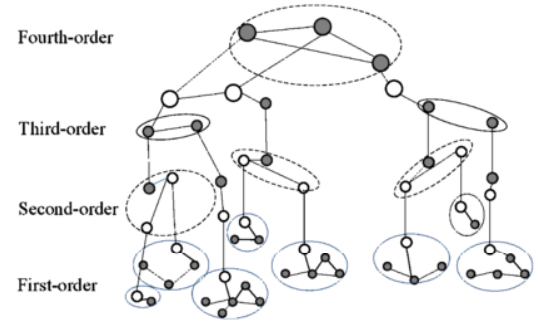

(b)

Figure 2. (a) Relation of the machines used for machining a part; (b) A hierarchical product network 


\subsection{A complex network model of CPPS}

Considering the shop floors in advanced manufacturing companies, they are characterised by intelligence, adaptive decision-making, and flexibility. To have an insight of the process of modelling the complex network within the context of CPPS, a complex network of the CPPS used for producing a product is shown in Figure 3, which reflects the layout of the shop floor and the machining process of the product.

The built-in sensing devices in this cyber-physical-enabled shop floor can sense and collect the real-time data of the manufacturing resources when they enter into the shop floor. Under this sensing framework, the RFID readers can sense and record RFID tags attached on the manufacturing resources, and then information of the manufacturing resources can be collected such as location, current status, and current machining process. Meanwhile, the production exceptions and machine failures can also be sensed which can be defined as RFID events. The workflow of collecting RFID events in the whole shop floor is shown in 'RFID events' in Figure 3. In the production \& assembly, the status of operators, materials, machines is recorded and checked. The machining execution of the production and assembly is then finished and the WIP (work in process) is sent to the buffer. The product raw material module is responsible for tracking and monitoring the status of the raw materials and the related manufacturing resources such as the pallet. Then, the complex network of the cyber-physical-enabled shop floor is presented and this network consists of 9 machining processes and assembly processes. Machining processes are $\mathrm{P}_{111}, \mathrm{P}_{112}, \mathrm{P}_{121}, \mathrm{P}_{122}, \mathrm{P}_{211}, \mathrm{P}_{212}$, and assembly processes are $\left\{\mathrm{P}_{A 1 a}, \mathrm{P}_{A 1 b}\right\} \rightarrow \mathrm{P}_{A 1},\left\{\mathrm{P}_{A 21}\right\} \rightarrow \mathrm{P}_{A 2},\left\{\mathrm{~F}_{A 2}, \mathrm{~F}_{A 2}\right\} \rightarrow \mathrm{F}_{A}$. According to the machining processes from the raw material area to final product area, it is obvious that the topology of the network can be highly complex. The flow of the machining process is $\mathrm{P}_{111} \rightarrow \mathrm{P}_{112}, \mathrm{P}_{121} \rightarrow \mathrm{P}_{122}, \mathrm{P}_{211} \rightarrow \mathrm{P}_{212},\left\{\mathrm{P}_{A 1 a}, \mathrm{P}_{A 1 b}\right\} \rightarrow \mathrm{P}_{A 1},\left\{\mathrm{P}_{A 21}\right\} \rightarrow \mathrm{P}_{A 2},\left\{\mathrm{~F}_{A 2}, \mathrm{~F}_{A 2}\right\} \rightarrow$ $\mathrm{F}_{A}$. When the exceptions or failures (overload) happen, the products and machines on three production lines perform the load reallocation. Failure nodes reallocate the load based on the proposed load allocation rules, which will be introduced in the following sections. From Figure 3, $\mathrm{P}_{111} \leftrightarrow \mathrm{P}_{121}, \mathrm{P}_{111} \rightarrow \mathrm{P}_{122}, \mathrm{P}_{112} \leftrightarrow \mathrm{P}_{122}, \mathrm{P}_{121} \leftrightarrow \mathrm{P}_{211}, \mathrm{P}_{122} \leftrightarrow$ $\mathrm{P}_{212}$ can perform the load reallocation in case of node failure. If the load of a node that accepts extra load does not exceed its load capacity under the load reallocation rules, and the growth and reconnection of the nodes and edges are performed, the load reallocation is then completed. Otherwise, the load reallocation of the failure nodes starts a new optimisation cycle until finished. This procedure of the load allocation is defined in the cascading failures/exceptions module. However, this process can lead to the cascading failure of the network nodes and even result in the breakdown the part of or the whole network. The load allocation rules and the evolution mechanism of the cascading failure are of great importance to explore the evolution of the cyber-physical-based complex network. 


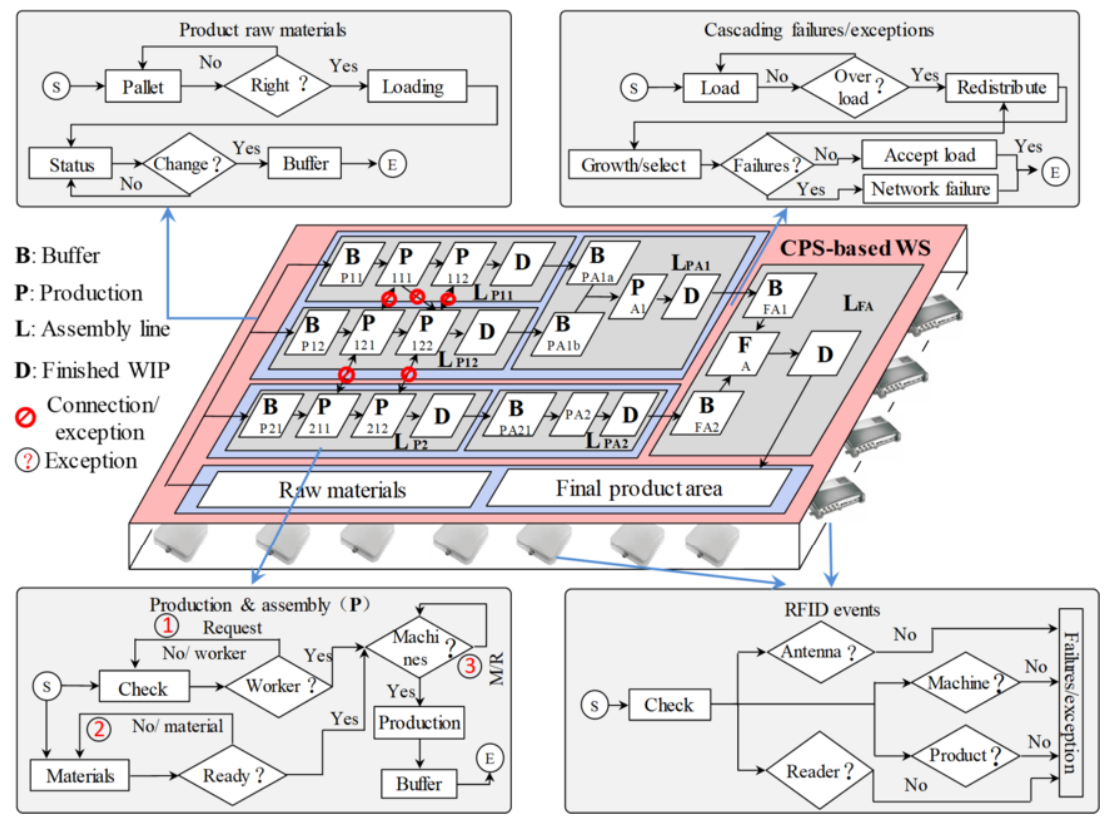

Figure 3. A network-based cyber-physical production system model

\section{Evolutionary mechanism of the cyber-physical-based complex network within the cascading failure}

The mechanism of the cascading failure of the complex network has recently gained a lot of attention in the field of manufacturing and is focused on the common-cause failure, load failure, and dependence failure. According to the definition of the cascading failure [21], the focus of work is given to an overload failure mechanism. The network in the real world has a certain form of 'flow' which produces the load. The load of the network can be reflected as two aspects: (1) node's load, the load capacity of the network node; (2) edge's load, the load capacity of the network edge.

The nodes in the machine and product networks are characterised by the transmission and distribution of the load. Each node or edge in the network has a certain load and capacity in which the capacity is the maximal load of the node or edge can handle. The nodes in the machine network have the machining load and capacity and can be in failure due to the exceptions such as machine breakdown, program errors, and machining errors. In the load distribution of the node, the load accepted by other nodes exceeding it capacity may happen, and this will lead to new load distribution of nodes and spread in the whole network, and finally result in the cascading failure of the nodes. Here, the complex network model within cascading failures under the neighbour preferential allocation rules is developed. 


\subsection{Cascading failure within the neighbour preferential allocation rules}

The load of the nodes for which the node failure happens is allocated to the part or all of the nodes in the network, and these key events induce the updating load of the nodes to accept the load of failure nodes. The exceeding load capacity of the node due to accepting a new load of the failure nodes triggers the new load allocation. Otherwise, the node works in the time step. The cascading failure of the network are addressed until the load of all the nodes of the network does not exceed their capacity.

The original load $L_{i}$ of node $i$ is defined by a function of the degree of the node $k_{i}$ and adjustable parameters of the load $(\beta, \omega)$. Then, the capacity of node $i\left(C_{i}\right)$ is proportional with its original load $L_{i}$, and it is formulated as a function of $L_{i}$ and a capacity coefficient $e$ which represents the ability of handling the extra load. When $e \gg$ 0 , any of nodes does not trigger the cascading failure. It has a finite value. The analysis of the network topology and evolution show that the machine and product networks are characterised by Barabási-Albert (BA) scale-free network [22]: preferential attachment and growth. Therefore, the scale-free network model is adopted in this paper.

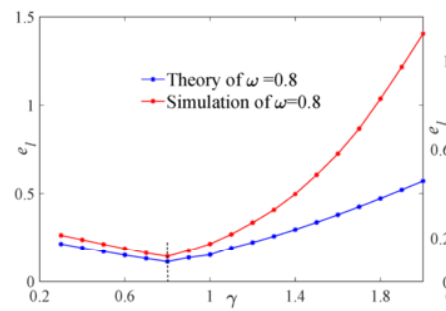

(a)

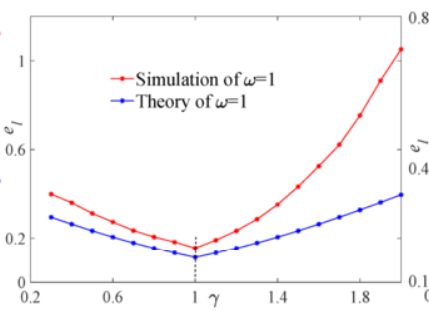

(b)

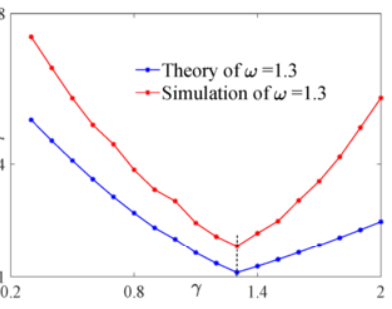

(c)

Figure 4. Analytical results for the BA scale-free network (a) $\omega=0.8$; (b) $\omega=1.0$; (c) $\omega=1.3$

To clarify the function of the intensity parameter of the original load $(\omega)$, the uniformity of the load distribution $(\gamma)$, and the threshold of the capacity efficiency $\left(e_{l}\right)$, three cases of $\omega$ are discussed, namely $\omega>1, \omega<1$, and $\omega=1$. Then, it is concluded that when $\omega$ is constant, $\gamma=e_{l}$ enables the complex network to have the strongest ability to resist cascading failures. The larger the average degree of the BA scale-free network $\langle k\rangle$ is, the larger the scale of the network is, and also the higher the ability to resist the cascading failure is. The analytical results of $e_{l}$ for the BA scale-free network are shown in Figure 4.

The complex network of CPPS has the typical characteristics of BA scale-free network which are preferential attachment and growth, and the degree distribution yields an approximately power-law distribution. Therefore, it can be described by the cascading failure model and the BA scale-free network. For the complex-network-enabled CPPS, the results show that the connection density of the network and the scale of the network contribute to resisting the cascading failure of the network. The increase in the capacity of the network can also improve the ability of resisting the cascading failure. However, these strategies are limited by cost and technical issues. The capacity of the network is finite. The original load of the node in the network and the allocation strategy of the nodes with the failure can affect the cascading failure of the network. When both $\omega$ and $\gamma$ are near one, the possibility of inducing the cascading failure in the network is the lowest. When the complex network is in stable status, $\omega$ and $\gamma$ tend to be the same, the possibility of causing the cascading failure is minimal. These analytic results contribute to modelling a robust complex network of CPPS. 


\subsection{Evolutionary mechanism of the importance of nodes within a cascading failure}

The study on the node importance of the network makes it possible to identify key nodes and edges which is of importance to improve the ability of resisting the cascading failure. Then, the cascading failure or the failure of the whole network induced by the breakdown on the key nodes and edges can be avoided. The BA scale-free network proposed in the above section has a better fault-tolerant ability to the random attack compared with the random network, while it shows the vulnerability of the network resisting the cascading failure.

In this section, the generation function-based identification scheme of the node importance is proposed. Then the importance of the node with the cascading failure can be determined and the key nodes in the network can be identified. Change from the key nodes to non-key nodes indicates the change in the ontology and initial status of the network and it also indicates the change from the steady status to the non-steady status of the network. Therefore, the procedure of identifying the node importance are introduced as follows:

1. Define the largest cluster. It is assumed that the network $V_{i}$ is composed of $N_{i}$ nodes and then $V_{i}$ has $N_{i}$ cluster $J_{i}\left(i=N_{i}\right)$, the largest cluster is the cluster with the largest number of nodes.

2. Define the possibility coefficient of the cluster in the network. The possibility coefficient $\theta\left(J_{i}\right)$ is the ratio of the number of the nodes within the cluster $J_{i}\left(N\left(J_{i}\right)\right)$ to that of the nodes in this network $\left(N_{i}\right)$.

3. Define the node importance. It is defined as the effect of deleting this node on the network such as the level of the damage.

4. Measure the importance of the node. Network $V_{i}$ is divided into multiple clusters $J_{k}(k \geq 1)$ after deleting node $i$. The possibility coefficient of the clusters is calculated and the node importance is formulated as $d_{i}=$ $\max _{k}\left(\theta\left(J_{k}\right)\right), J_{k} \subset V_{i}$.

5. Identify the key nodes or key node sets. The importance of all the nodes in the network is calculated and ranked in an ascending order. The node with the possibility coefficient of the cluster lower or equal to the value of the key node is defined as a key node, namely $I_{k e y}=I\left(d_{i}\right)$. If they are multiple nodes, they are defined as a key node set $\left\{I_{\text {key }}\right\}=I_{\text {key }}\left(d_{j}<d_{i}\right)$.

The proposed identification method of the key nodes can avoid the drawbacks induced by: 1) the importance is equal to the damage of deleting the node on the network, and 2) the importance is equal to the significance. The proposed method also considers the structure of the network, the spreadability and mechanism of the network behaviour, and the properties of the node.

Then, the importance of node $i(D(i))$ is defined as the average fluctuation degree of all the nodes in the range of the parameter of the load allocation and is formulated as (1). Details of the derivation procedure are omitted here due to space constraints.

$$
D(i)=\sum_{j \in \Omega_{i}} d_{i j}^{-\lambda} k_{j}^{\gamma-\omega} k_{i}^{\omega} /\left[N\left(\Omega_{i}\right) e \sum_{n \in \Omega_{i}} d_{i n}^{-\lambda} k_{n}^{\gamma}\right]
$$

where $N\left(\Omega_{i}\right)$ is the number of the nodes in $\Omega_{i}$. If $D(i) \geq 1$, the failure of node $i$ must induce the cascading failure by the overload of the node. If $D(i)<1 / N\left(\Omega_{i}\right)$, the failure 
of node $i$ does not trigger the cascading failure. If $1 / N\left(\Omega_{i}\right) \leq D(i)<1$, the failure of node $i$ has the uncertainty on the induction of the cascading failure. $\lambda \in[0, \infty]$ and $\gamma \in$ $[0, \infty], \lambda \rightarrow 0$ indicates the global preferential allocation rules, and $\lambda \rightarrow \infty$ indicates the neighbour preferential allocation rule. Here, due to the characteristics of the complex network of CPPS: preferential attachment and growth, the effort is given to the case of $\lambda \rightarrow \infty$. Then, the analytical function of the node importance of the BA scale-free network with the neighbour preferential allocation rule is formulated as follows:

$$
\begin{aligned}
& \quad D(i)^{S F}=\left[k_{i}^{\omega-1}(\gamma-r+2) 2^{\omega}\left(N^{[\gamma-\omega-r+2] /[r-1]}-1\right)\right] /[e(\gamma-\omega-r+2)< \\
& \left.k>^{\omega}\left(N^{[\gamma-r+2] /[r-1]}-1\right)\right]
\end{aligned}
$$

It can be derived from (2) that the safety and risk thresholds are $1 / N\left(\Omega_{i}\right)$ and 1 . $D(i)<1 / N\left(\Omega_{i}\right)$, the failure of node $i$ does not induce the cascading failure; $D(i) \geq 1$, the failure of node $i$ leads to the overload-induced cascading failure. Therefore, when the importance of all the nodes in the network is lower than the safety threshold, then the network can handle the failure of all the nodes without inducing the cascading failure. When the importance of the key node is larger than the risk threshold, the failure of the node induces the overload of other nodes; The importance of the node with the least importance is larger than the risk threshold, the failure of any node must induce the breakdown of the whole network. The lower bound of the capacity coefficient of these three cases are defined as $e_{1}, e_{2}$, and $e_{3}$. Figure 5 shows the lower bound of the capacity coefficient of these three kinds of the networks.

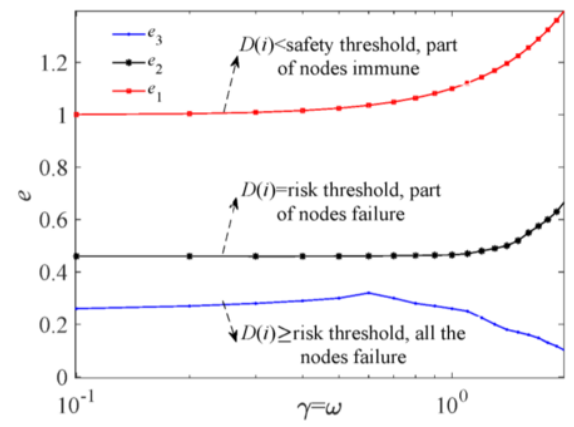

Figure 5. Lower bound of the capacity coefficient of the network

From Figure 5, the area of resisting the cascading failure of the network is located in the area with a larger value of $e_{1}$ and the importance of all the nodes is lower than $1 / N\left(\Omega_{i}\right)$. The area with the value smaller than $e_{3}$ is the total cascading failure area and the failure of any node in the network can induce the breakdown of the network. The area with the value between $e_{1}$ and $e_{2}$ is the random failure area and the importance of all the nodes is in the range of $1 / N\left(\Omega_{i}\right)$ and 1 . The area with the value between $e_{2}$ and $e_{3}$ is the failure of some nodes can induce cascading failure and the importance of some nodes is larger than 1 .

Then, the effect of $e, \omega$, and $\gamma$ on the importance of the node in the BA scale-free network is studied. $M=1000, N=3, N_{0}=2,<k>=6$, and the importance of each node is assumed to be the average of the importance of the same nodes. Figure 6 shows the relation of the node importance $\left(d_{i}\right)$ and the parameters for BA scale-free network within the neighbour preferential allocation rule: a) with the capacity coefficient $(e)$; b) with the 
intensity parameters of the original load $(\omega)$; c) with the uniformity of the load allocation $(\gamma)$.

Figure 6(a) shows the capacity coefficient large enough to enable any node in the network not induce the cascading failure within the neighbour preferential allocation rule. Meanwhile, the importance of the node only reflects the structure importance of the node and the distribution of the importance of the node is an approximately exponential function with the importance degree. From Figure 6(b), the power exponent of the distribution of the node importance is related to the intensity parameter of the original load. Under the neighbour preferential allocation rule, (1) $\omega>1$ and $r>0$ indicates that the degree of the node is proportional with the importance of the node; (2) $\omega<1$ and $r<0$ indicates the node with a low degree is a potential key node. From Figure 6(c), $\gamma$ is inversely proportional to the possibility of inducing the cascading failure. The node with the uneven load distribution has a low possibility of happening the cascading failure. The non-uniformity is reflected on the node with large degrees having a large load. Therefore, by analysing the problem of the effect of the non-key nodes on the cascading failure, it can be concluded that the intensity parameter of the original load and the uniformity parameter of the load distribution are two key factors influencing the importance evolution of the nodes for BA scale-free network.

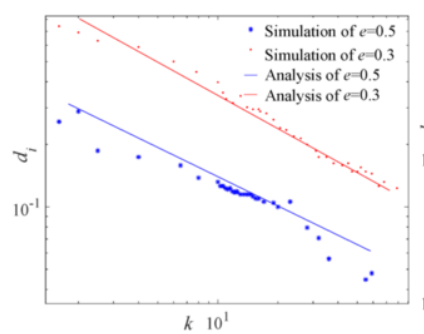

(a)

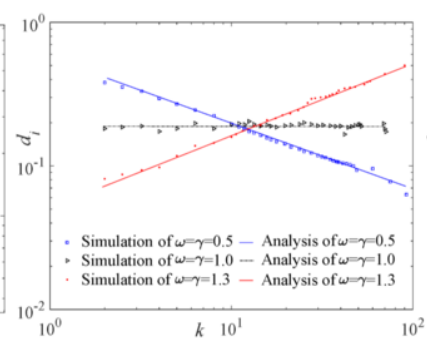

(b)

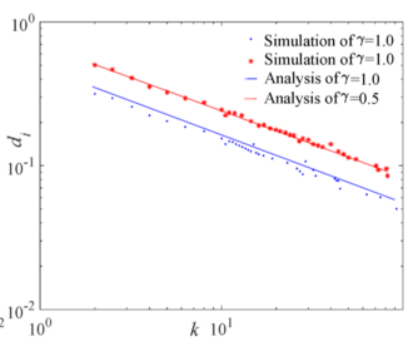

(c)

Figure 6. Relation of $d_{i}$ and: a) $e$; b) $\omega$; c) $\gamma$ within the neighbour preferential allocation rules

\section{Conclusions}

This paper studies the cascading failure of a complex network-based cyber-physical production system. Through the use of IoT, the evaluation of the reliability importance of individual nodes is monitored to address the operating challenges arising in complex manufacturing systems. The contributions of the study are summarised as follows. First, our method is based on real-time, event-driven information sensing and capturing method. The proposed CPPS is capable of sensing real-time manufacturing states and catching production exception processes via the embedded sensor network. Second, local load preferential allocation rule is employed to build the cascading failure model. It allows for exploring the self-organisation evolvement of CPPS, and further deriving the propagation path of cascading failure. Third, the evolution of nodes' reliability importance enables us to track how a non-key node evolves into a key node over the time under the overload mechanism. Our future work will focus on advanced algorithm development for real-world complex industrial practices. 


\section{Acknowledgement}

This work is funded by EUREKA SMART: Cyber-Physical Assembly and Logistics System in Global Supply Chains (C-PALS).

\section{References}

[1] L. Wang, "From Intelligence Science to Intelligent Manufacturing," Engineering, vol. 5, no. 4, pp. 615-618, 2019.

[2] L. Wang, X. Xu, R. Gao, and A. Y. C. Nee, "Sustainable cybernetic manufacturing," Int. J. Prod. Res., vol. 57, no. 12, pp. 3799-3801, 2019.

[3] L. Wang, M. Törngren, and M. Onori, "Current status and advancement of cyber-physical systems in manufacturing,” J. Manuf. Syst., vol. 37, no. April 2016, pp. 517-527, 2015.

[4] E. a Lee, "Cyber-Physical Systems - Are Computing Foundations Adequate?," October, vol. 1, no. January 2006, pp. 1-9, 2006.

[5] L. Monostori et al., "Cyber-physical systems in manufacturing," CIRP Ann., vol. 65, no. 2, pp. 621641, Jan. 2016.

[6] L. Wang, X. V. Wang, L. Wang, and X. V. Wang, "Latest Advancement in CPS and IoT Applications," Cloud-Based Cyber-Physical Syst. Manuf., pp. 33-61, 2018.

[7] L. Wang and A. Haghighi, "Combined strength of holons, agents and function blocks in cyber-physical systems,” J. Manuf. Syst., vol. 40, pp. 25-34, 2016.

[8] Y. Zhang et al., "The 'Internet of Things' enabled real-time scheduling for remanufacturing of automobile engines," J. Clean. Prod., vol. 185, 2018.

[9] S. Liu, G. Zhang, and L. Wang, "IoT-enabled Dynamic Optimisation for Sustainable Reverse Logistics," in Procedia CIRP, 2018, vol. 69.

[10] S. Liu, Y. Zhang, Y. Liu, L. Wang, and X. V. Wang, “An 'Internet of Things' enabled dynamic optimization method for smart vehicles and logistics tasks," J. Clean. Prod., vol. 215, 2019.

[11] Y. Zhang, S. Liu, S. Si, and H. Yang, "Production system performance prediction model based on manufacturing big data," in ICNSC 2015 - 2015 IEEE 12th International Conference on Networking, Sensing and Control, 2015.

[12] G. Q. Huang, Y. F. Zhang, and P. Y. Jiang, "RFID-based wireless manufacturing for real-time management of job shop WIP inventories," Int. J. Adv. Manuf. Technol., vol. 36, no. 7-8, pp. 752-764, 2008.

[13] Y. Zhang, W. Wang, S. Liu, and G. Xie, "Real-time shop-floor production performance analysis method for the internet of manufacturing things," Adv. Mech. Eng., vol. 2014, 2014.

[14] Y. F. Zhang and T. F, Optimization of Manufacturing Systems Using the Internet of Things. 2016.

[15] Y. Li, Y. Cheng, X. Zhang, and A. Y. C. Nee, "Complex networks in advanced manufacturing systems,” J. Manuf. Syst., vol. 43, pp. 409-421, Apr. 2017.

[16] Y. Cheng, F. Tao, L. Xu, and D. Zhao, "Advanced manufacturing systems: supply-demand matching of manufacturing resource based on complex networks and Internet of Things," Enterp. Inf. Syst., vol. 12, no. 7, pp. 780-797, 2018.

[17] I. Dobson, B. A. Carreras, V. E. Lynch, and D. E. Newman, "Complex systems analysis of series of blackouts: Cascading failure, critical points, and self-organization," Chaos, vol. 17, no. 2, 2007.

[18] B. Wang and B. J. Kim, "A high-robustness and low-cost model for cascading failures," Epl, vol. 78, no. 4, 2007.

[19] P. Li, B. H. Wang, H. Sun, P. Gao, and T. Zhou, "A limited resource model of fault-tolerant capability against cascading failure of complex network," Eur. Phys. J. B, vol. 62, no. 1, pp. 101-104, 2008.

[20] Y. Zhang, W. Wang, N. Wu, and C. Qian, "IoT-Enabled Real-Time Production Performance Analysis and Exception Diagnosis Model," IEEE Trans. Autom. Sci. Eng., vol. 13, no. 3, pp. 1318-1332, 2016.

[21] P. Crucitti, V. Latora, and M. Marchiori, "Model for cascading failures in complex networks," Phys. Rev. E - Stat. Physics, Plasmas, Fluids, Relat. Interdiscip. Top., vol. 69, no. 4, p. 4, 2004.

[22] G. Bianconi, "Mean field solution of the Ising model on a Barabási-Albert network," Phys. Lett. A, vol. 303, no. 2-3, pp. 166-168, Oct. 2002. 